\title{
Digital Twinning of Railway Overhead Line Equipment from Airborne LiDAR Data
}

\author{
M.R.M.F. Ariyachandra ${ }^{1}$ and Ioannis Brilakis ${ }^{2}$ \\ Department of Engineering, University of Cambridge, United Kingdom \\ E-mail: mfa47@cam.ac.uk, $\underline{\text { ib340@cam.ac.uk }}$
}

\begin{abstract}
The automated generation of geometry-only digital twins of Overhead Line Equipment (OLE) system in existing railways from point clouds is an unsolved problem. Currently, this process is highly reliant upon manual inputs, needing 10 times more labour hours than scanning the physical asset. The resulting modelling cost counteracts the expected benefits of the digital twin. We tackle this challenge using a novel model-driven method that exploits the highly regulated and standardised nature of railways. It starts by restricting the search for OLE elements relative to point clusters of the railway masts. The resulting point clusters of the OLE elements are then converged with various parametric models of different catenary configurations to verify the presence of OLE elements and to find the best possible fit. The method outputs a geometry-only digital twin of the OLE system in Industry Foundation Classes (IFC) format. The method was tested on an $18 \mathrm{~km}$ railway point cloud and achieves overall detection rates of $93.2 \%$ F1 score for OLE cables and $98.1 \%$ F1 score for other OLE elements. The accuracy of the generated model is evaluated using distance-based metrics between the ground truth model and the automated model. The average modelling distance is $3.82 \mathrm{~cm}$ Root Mean Square Error (RMSE) for all $18 \mathrm{~km}$ data.
\end{abstract}

Keywords -

Overhead Line Equipment (OLE); Geometric Digital Twin (gDT); Point Cloud Data (PCD)

\section{Introduction}

Cost overruns are a worldwide phenomenon for railway projects, irrespective of the size and the contract value. The average cost overruns account for $29 \%$ projects' engineer estimates for rail and road projects in Europe and the United Kingdom (UK) [1], [2]. For example, the London Docklands Light Rail project is currently overrunning by over $£ 1$ billion in costs and three years [3] in duration. These cost and time overruns are common partly due to the absence of Information and communications technology (ICT) sector-level data management for construction/upgrade and maintenance of railways. Specifically, the absence of any form of a digital representation of railways caused by the extensive time required for collecting and processing raw data into working models makes it challenging to upgrade railways.

We argue that the need to create and maintain up-todate digital twins of railways is an opportunity that should not be missed. Digital twins are expected to bring significant benefits to time, cost and quality parameters of railway projects [4]. These benefits include an $80 \%$ reduction in time, $10 \%$ through clash detection and $40 \%$ elimination of unbudgeted change [5], [6]. Yet, the current cost of creating and maintaining the digital twins greatly counteracts the perceived benefits of the digital twin. The non-canonical shapes in railways require $95 \%$ of the total modelling hours for manual shape customisation and fitting processes [7]. The automation of the twinning process will reduce the modelling time and ultimately save costs. We presented a method for generating railway masts as a first step to tackling this challenge [8], which also presented a method for removing the majority of the vegetation and other noise data from the input Point Cloud Data (PCD). The current paper addresses the next step; a method to automatically generate Geometric Digital Twin (gDT)s of railway Overhead Line Equipment (OLE) systems from airborne LiDAR data.

\section{Overhead Line Equipment (OLE)}

We define the following components as OLE elements (Figure 1).

- Connecting beam - Connects two masts of the same pair together.

- Contact cable - Transmits power to the train by a pantograph. They lie in the lowest height among all overhead cables and shall not be placed less than $5.8 \mathrm{~m}$ from the ground [9]. The contact cable runs in a zig-zag path above the track to avoid wearing a 
groove in the pantograph. The zigzag known as the 'stagger' - is generally achieved using 'pull-off' arms attached to the support structures.

- Auxiliary cable - Placed between the catenary and contact cables, functions as a buffer and reduces the fluctuation of the cables.

- Catenary cable - Supports the contact cable and located immediately above auxiliary cables.

- Cantilever - Consists of horizontal and vertical metal tubes, connects catenary cables to masts. It supports the catenary projecting from a single mast on one side of the track.

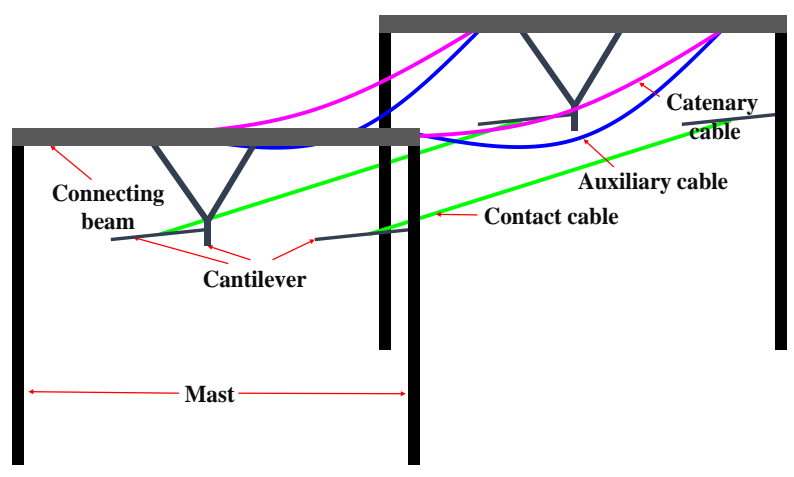

Figure 1. OLE elements

\section{Research Background}

\subsection{Contact, Auxiliary and Catenary Cables}

The process of converting PCD into gDTs of cables involves three steps: (1) Extraction of cable points, (2) Clustering single cable points, and (3) 3D cable fitting. Methods for cable extraction and clustering include: (1) Statistical analysis of PCDs based on height, density or number of pulses, etc. [10]-[14] (2) Hough transform and clustering based on two-dimensional (2D) image processing [11], [15], [16] (3) Supervised classification based on metrical and distribution features between points [13], [17]-[19]. Verification of detected cables is performed using a simplified model to fit into detected cables in the planar horizontal view [20] or by the usage of the Random Sample Consensus (RANSAC) algorithm combined with intensity values [21] or a polynomial function to fit the model [22]. We review each of these for cables in both railways and roads.

\subsubsection{Statistical Analysis of PCDs Based on Height, Density or Number of Pulses}

Jwa and Sohn [23] converted the cloud into voxels and detected cables using the linearity of the element. The method also used point density and segmentation analysis to differentiate roof edges, fences and other linear assets from cables and to group the points into catenary and contact cables. However, their method highly depended on the point density and the size of the voxel. The method did not work well when the cloud had data gaps, vegetation encroachment, and bundled cables. Cheng et al. [10] used a similar approach following $\mathrm{KD}$ trees and a polynomial function for clustering and 3D model fitting. The main limitation was they ignored the sagging posture of cables. Also, the method was influenced by frequent occlusions by trees or buildings, ambient conditions such as the temperature and the ageing of spans. The high detection and clustering accuracies were also attributed to the high point density of the data.

Zhu and Hyyppa [11] used statistical analysis considering height, point density and histogram thresholds and image processing methods to extract cables considering geometric properties. However, the thresholds highly relied on the point density and their dataset had a clear cut-off edge between cables and trees. In the majority of cases, OLE cables are not located at a distance away from the surrounding trees and other pole-like objects. A similar approach was used in Guan et al [14] to differentiate road and off-road points and to extract power-transmission cables/power tower points from the latter, followed by the extraction of individual power-transmission cables via Hough transform and Euclidean distance clustering. Finally, a 3D object fitting was done using linear and hyperbolic cosine functions. However, the method was sensitive to the point density of their PCD and did not work well for a different point cloud. Following previous methods, Cserép et al. [13] used height analysis to filter ground points and then used intervals along a selected axis with a point counter assigned to each interval to remove outliers. Finally, the method extracted cable points using using a density analysis and 3D voxels. Yet, their method could not reconstruct those cables into 3D models as the density algorithms removed some cable points as well.

\subsubsection{Hough Transform, and Clustering Based on 2D Image Processing}

Liu et al. [15] used statistical analysis and an improved Hough transform [24], to segment and to detect cable points. However, the thresholds were sensitive to the point density of the PCD and further work is needed to map the detection results to original 3D data and fit the curves to cable points. The method used in Sohn, Jwa and Kim [16] segmented cable points using Markov Random Field (MRF) classifier, which classifies power cables from other linear assets. The locations of pylons were used to detect cable span, within which cables are modelled with catenary curve models in 3D using the piecewise model growing. Yet this method assumed that the cables were parallel. 


\subsubsection{Supervised Classification Based on Metrical and Distribution Features between Points}

Kim and Sohn [17] used Random Forests classifier as a supervised classification method to detect five key object classes which include cables, pylons, building, vegetation and low objects. They used 2D Hough Transform to 2D points of cables obtained by projecting 3D points onto a horizontal plane. A similar approach has been used in Wang et al. [19] by filtering cables using the height relative to the other objects. Then, the multi-scale spherical neighbourhoods are used to capture the anisotropy and details of cable topology structure. Next, metrical and distribution features were used to enhance classification accuracy. Finally, using Support Vector Machine (SVM) they classified cable points. However, both methods were unable to perform well when cables become the only object of interest as all the feature-based classifiers were highly attributed to the five class objects they considered. Furthermore, these methods only classified but not reconstructed 3D models of any of the class objects.

Following the same classification process, Guo et al. [18] used an improved RANSAC algorithm that includes similarity detection. A powerline between a span was segmented into neighbourhood cubes. Then the points within the cubes were projected onto planes to detect the similarities amongst the planes. At last, they detected cables using the inliers selected by RANSAC. However, the accuracy of the method was sensitive to the sparseness of the data and did not work well for a different point cloud. For instance, if there are few or no points on the cables, data are classified as vegetation by default. Also, cables were split into several pieces or categorised as false negatives when parts of the cables are being obscured by vegetation or when there were few points along a section of the line.

\subsection{Cantilevers and Connecting beams}

There are only two methods exist that detect cantilevers from PCD in Pastucha [25] and Rodríguez et al. [26] and no methods exist that detect connecting beams. In Pastucha [25], the method used the pattern of the points; a cross above the track to indicate the presence of cantilevers using RANSAC algorithm. All model points are classified as the catenary system in the case of positive authentication. Yet, the high detection rates were attributed to the trajectory of the mobile scanner and the density of the PCD. Besides, all the geometrical distances and properties of the objects should be manually entered by the end-user. Rodríguez et al. [26] detected points of cantilevers using a range search algorithm to filter the highest set of points relative to the points on the catenary cable. However, they could not validate the method since the data set was too small, so it only contained one cantilever.

\subsection{Gaps in Knowledge}

Cable scene complexity including data gaps, vegetation encroachment [11], [18], and bundled cables affected the accuracy of the results of the existing OLE elements detection methods [10], [23], [26]. Majority of methods were sensitive to the size of the voxels used [23], the setting of thresholds, point density [10], [11], [14], [15], [18] and to ambient conditions such as the temperature and the ageing of spans [10]. A few studies disregarded the sagging of the cables [10], while some assumed cables as a set of small straight-line segments [13], [23] and that cables are always parallel [16]. These methods were unable to distinguish cables from other straight lines such as building roof edges, fences and tree stems [23]. Also, these methods did not perform well when reconstructing 3D models of OLE elements as the initial filtering removed some of the OLE elements points as well [13]. Finally, one of the methods used to detect cantilevers considered a very small dataset which only contains one cantilever and the method did not contain any validation process [26]. OLE elements are very thin, hence often represented with few or no points. The detection of OLE elements is a very hard problem also due to the presence of vegetation. These factors render the methods discussed for OLE elements detection ineffective. Also, the gDT generation for OLE systems in existing railways is almost missing in the literature.

\section{Proposed Solution}

\subsection{Scope}

Our method focuses on typical double-track railways that represent $70 \%$ of the existing and under construction railways in the UK [27]. Railways are a linear asset type; therefore, their geometric relations remain roughly unchanged often over very long distances. Close inspection of railway PCD validates this effect, with repeating geometrical features such as; (1) the special relations between masts, cables and the rails remain fairly unchanged along the railway [9], (2) the connections between masts and the cables are placed in regular intervals (roughly 50-70m intervals), (3) the main axis of the masts (Z-axis) is roughly perpendicular to the track direction (X-axis) (error tolerance is $11^{\circ}$ [9]) and (4) masts are always positioned as pairs throughout the rail track.

\subsection{Overview}

It is often the case that there are few to no points on the OLE elements in a railways PCD due to the small 
size of the cables compared to the size of the asset. This is expected and likely to occur no matter what scanning technology is used. This creates obstacles that hinder the robustness of OLE elements reconstruction. We argue that the strengths of model-driven strategies (providing fast geometrical models without visual deformations [28]) are strong in the scenarios with very low point densities meaning the model-driven methods can create the gDTs of OLE elements despite poor densities and sparseness of the PCD. Hence, our method proposed a fitting of pre-defined parametric assemblies to the point segments obtained using previously sorted mast position coordinates [8]. This proposed method is a re-iterative method throughout the track; it exploits the railway geometric relations mentioned in section 4.1 and railway topology as key factors. The workflow of the proposed method is illustrated in Figure 2.

\begin{tabular}{|c|c|c|}
\hline \multicolumn{2}{|l|}{ START } & $\begin{array}{c}\text { Mast position coordinates }+ \text { Narrowed } \\
\text { railway PCD }\end{array}$ \\
\hline & \multicolumn{2}{|c|}{$\begin{array}{l}\text { Step 1: Extract point clusters of } C \text { sections of OLE } \\
\text { elements (Masts, Connecting beam and Cantilever) } \\
\text { Step 2: Extract point clusters of cables } \\
\text { Step 3: Generate dynamic IFC models of OLE elements }\end{array}$} \\
\hline \multicolumn{2}{|c|}{ Input/Output } & $\begin{array}{c}\text { Model A-Point clusters of OLE elements } \\
\text { Model B-IFC OLE elements }\end{array}$ \\
\hline & \multicolumn{2}{|c|}{$\begin{array}{l}\text { Step 4: Convergence of Model A and Model B; } \\
\text { i. Sort the correct version (left or right) of the model B } \\
\text { ii. Align the sorted Model B in the correct position } \\
\text { iii. Get the transformation matrices }\end{array}$} \\
\hline \multicolumn{2}{|c|}{ Input/Output } & Model B + transformation matrices \\
\hline & \multicolumn{2}{|c|}{$\begin{array}{l}\text { Step 5: Use transformation matrices to move Model B } \\
\text { to the correct position + Merge all elements into one .ifc } \\
\text { file }\end{array}$} \\
\hline END & & IFC model of the OLE system elements \\
\hline
\end{tabular}

Figure 2. The workflow of the proposed method

\subsection{Extract Point Clusters of C Sections of OLE Elements - Model A}

\subsubsection{Point Clusters of Other OLE Elements - C Sections}

Initially, we extract point clusters of the OLE system includes two railway masts, the connecting beam and the cantilever. This unit is hereafter known as ' $\mathrm{C}$ section'. We use a passthrough filter to extract the point segment of the $\mathrm{C}$ section. The pass-through filter passes the input points through constraints based on a particular field. It iterates through the entire PCD once, performing two operations. First, it removes non-finite points. Second, it removes any points that lie outside the specified interval for the specified field.
In our proposed method we have set three fields, which refer to intervals along the $\mathrm{X}, \mathrm{Y}$ and $\mathrm{Z}$ directions. The limits are defined relative to the mast coordinates so that the filter removes any points according to the given direction and the limits. Specifically, the $\mathrm{X}$ range is experimentally set to $0.5 \mathrm{~m}$ in the direction along the track length. This range has been set relative to the $\mathrm{X}$ coordinates of masts to include the width of the mast allowing $0.2 \mathrm{~m}$ of a buffer window. The $\mathrm{Y}$ range is the distance between two masts of the same pair, calculated using $\mathrm{Y}$ coordinates of the two masts and the experimentally set buffer window of $0.5 \mathrm{~m}$, in the direction along the track width. The $\mathrm{Z}$ range is experimentally set to $9.5 \mathrm{~m}$ (height of the mast) in the direction along the mast. We have experimentally eliminated $0.2 \mathrm{~m}$ from the ground plane to remove ground points as it would suffice to extract the $\mathrm{C}$ section without shortening the mast. In this paper, we haven't illustrated the graphs representing calculations for these parameters due to limited space. This finally gives resulting point segments of $\mathrm{C}$ sections along the track.

\subsubsection{Point Clusters of Cables}

We use an improved RANSAC algorithm to extract point clusters of cables. The method starts by determining Bounding Boxes (BB) using mast position coordinates along the track to crop the input PCD such that the resulting pieces are relatively straight enough for any further processing. The general RANSAC could not detect cables as lines due to few or no points on the cables.

Hence, we initially up-sampled the points on cables to improve line detection. The up-sampling was done along the track direction (Figure 3) with defined intervals along either side of the actual points. The track direction is not constant along a particular direction due to the varying horizontal and vertical elevations along the track. To determine the track direction prior to upsampling, we calculate the range between minimum and maximum of $\mathrm{X}$ and $\mathrm{Y}$ values of each $\mathrm{BB}$ and sort the general track direction along the $\mathrm{X}$-axis if the $\mathrm{X}$ range > $\mathrm{Y}$ range and vice versa (Figure 4 ).

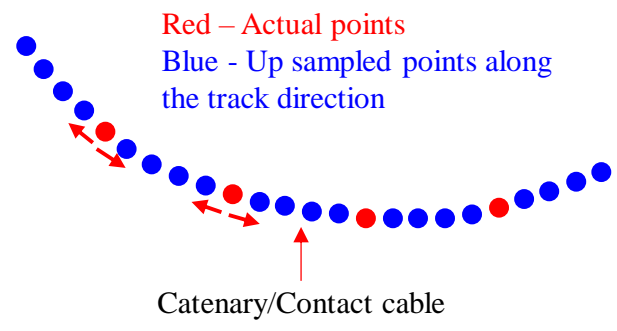

Figure 3. Upsampling along the track direction 


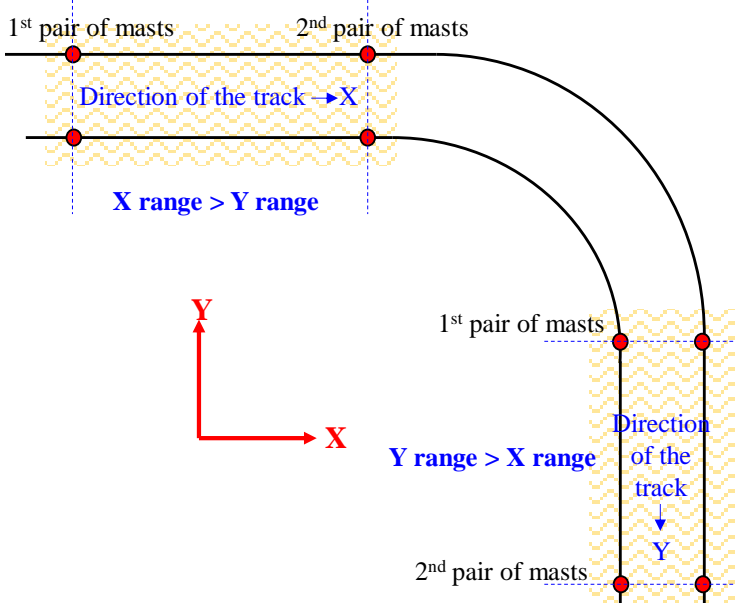

Figure 4. Determining general track direction

Next, we use two pre-processing steps prior to the RANSAC algorithm to improve line detection and to reduce the computational cost. Firstly, we remove the ground plane to eliminate all ground points. This ensures all points on the ground are removed prior to further calculations. This significantly reduces the points for faster computational performance and reduces the number of false positives that would be caused by the lines on the ground. Secondly, we get the XY projection of the cloud. This allows projecting the catenary shapes of the catenary and auxiliary cables into straight lines so that RANSAC can detect those cables despite its curved shape. We then detect cables as lines using RANSAC and classify cables based on the heights of the lines relative to the track structure. The detected cables along with the previously extracted $\mathrm{C}$ sections are hereafter known as 'Model A'.

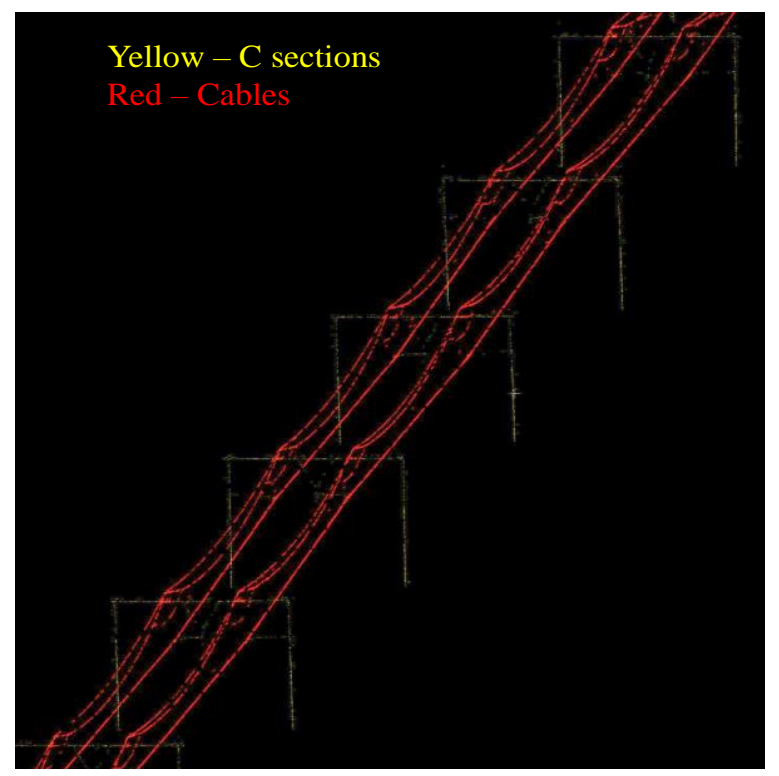

Figure 5. Point clusters of OLE elements - Model A

\subsection{Generate Dynamic IFC Models of the OLE System}

We design a parametric OLE system model; hereafter known as 'Model B' using standard railway electrification guidelines [9] to represent the geometry of the real OLE elements. This model preserves geometrical properties of the elements, such as angles between the different elements, relative distances compared to each element of the system. The model developed during this research is much simpler compared to the real OLE system as the model is limited only for the elements defined at the beginning of this paper. This limited number of elements simplifies the task of adjusting the model while the resulting model is still suitable to reconstruct the geometric shape of the OLE system.

The orientation of Model B constantly changes from left to right along the track due to the stagger occur in the OLE system. However, this alignment might change if the track is not perfectly straight so that we cannot assume the orientation of the model. Hence, we have created 10 variations of Model B, compatible with the left and right versions of the 5 types of the OLE configurations exist in UK railways [9]. Figure 6 illustrates only one of those configurations due to the limited space. Note that on the actual model, two of these configurations (from the same type) are connected with cables.

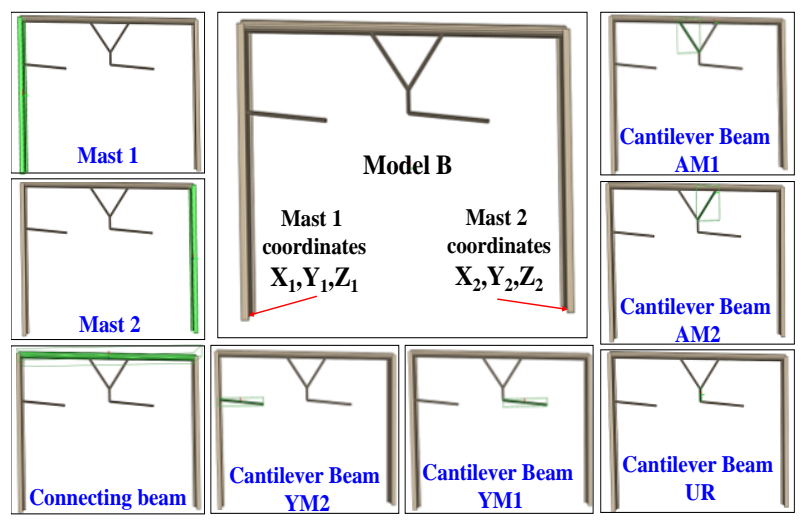

Figure 6. The left-to-right orientation of one of the OLE configurations

We define each of the OLE elements using extruded area solid definition in IFC format. We use the standard cross-sectional dimensions given on Network Rail standards [9] to define the 2D area profile for each element. The extruded area solid defined the extrusion of a $2 \mathrm{D}$ area; here defined as the section profile, by two attributes. One is the extruded direction, defining the direction in which the profile is to be swept. The other attribute is the distance over which the profile is to be swept. For each OLE element, we define these distances using either standard height (for masts) or length (for 
every other OLE element). The extruded direction and relative angles are derived considering the position and the orientation of each element relative to mast positions.

\subsection{Convergence of Model A and Model B}

Iterative closest point (ICP) algorithm aims to find the transformation between a point cloud and its reference cloud, by minimizing the square errors between the corresponding entities. We use this algorithm to converge Model B to Model A. Initially, we set Model A as the reference cloud; is kept fixed while the left and right orientations of Model B are source clouds. We first convert Model B into .pcd files and then these source clouds are transformed to find the best match with the reference - Model A. The ICP iteratively revises the transformation of Model B to minimize the distance to the Model A, such as the sum of squared differences between the coordinates of the matched pairs. Hence, by using ICP we first sort the correct orientation (left or right) of OLE configuration as the correct orientation ideally has the minimum sum of squared differences between the coordinates of the target and reference clouds. Once we sorted the correct orientation, our method then converges the sorted model on to the correct position and finally gives transformation matrix which provides the corresponding translation vector and rotation matrix of the Model B (model) relative to Model A (point cluster). Finally, we moved .ifc format of the Model B to the correct position using the resulting transformation matrices of the previous step and finally merged all units (including two $\mathrm{C}$ sections and cables) into one file to get the final IFC model of the OLE elements.

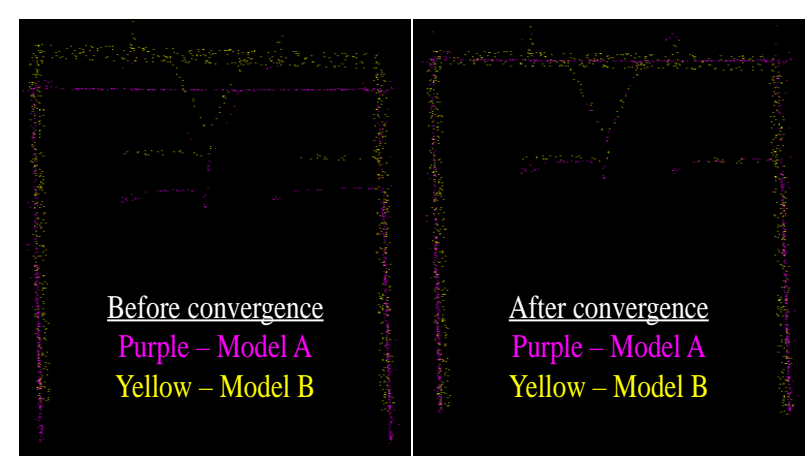

Figure 7. The convergence of Model A and Model B

\section{Experiments}

\subsection{Ground Truth Data and Results}

We used the rail track located in-between 'sHertogenbosch and Nijmegen in the Netherlands and specifically a piece of the railroad track that is approximately $18 \mathrm{~km}$ long, to test our proposed method. The size of this file was over $100 \mathrm{~GB}$ hence too large to process with the machines available in terms of processor and memory capacity. We address this challenge by splitting the data file into three sub PCDs as D1, D2 and D3 each length around $6 \mathrm{~km}$. We also manually generated two sets of Ground Truth (GT) datasets consist of three sub-datasets each per one railway $\mathrm{PCD}$;

GT A: This set is created by manually extracting point clusters of $\mathrm{C}$ sections and cables along the rail track. They are used to compare against the automatically detected point clusters of OLE elements.

GT B: The set is created by manually creating the OLE systems models. They are used to compare against automatically generated gDTs of OLE elements.

We implemented the solution with the Point Cloud Library (PCL) version 1.8.0 using C++ on Visual Studio 2017, in a laptop (Intel Core i7-8550U 1.8GHz CPU, 16 GB RAM, Samsung 256GB SSD).

Table 1 illustrates the results of the point cluster extraction and Figure 8 demonstrates the results of the automated gDTs compared to GT B. The detection of OLE elements needed an average of 35 seconds per $\mathrm{km}$. Generation of dynamic IFC models and conversions took 16 seconds per $\mathrm{km}$, while the convergence required 51 seconds per $\mathrm{km}$. Finally, the transformation only took 0.2 seconds per $\mathrm{km}$. Hence, the processing time of the proposed method was on average 103 seconds $/ \mathrm{km}$.

\section{Evaluation}

\subsection{Evaluation of point cluster extraction}

We used performance metrics; precision (Pr) and recall $(\mathrm{R})$ and $\mathrm{F} 1$ score $(\mathrm{F} 1)$ as; True Positive (TP) OLE elements were correctly detected as OLE elements, False negatives (FN) - OLE elements were not detected as OLE elements and False Positives (FP) - other objects were detected as OLE elements, to measure the performance step 1 and 2 . The average detection accuracy for $\mathrm{C}$ sections was $98.1 \% \mathrm{~F} 1$ score and for cables $93.2 \%$ F1 score (Table 1).

\subsection{Evaluation of the OLE system gDTs}

We used cloud-to-cloud (C2C) distance evaluation to detect changes between GT B and the automated ones. Initially we converted the GT B and the automated gDTs into .pcd files. Then we computed the Root Mean Square Error (RMSE) between each unit of automated gDT of OLE elements (consists of two $C$ sections and cables) and corresponding GT B model (Figure 8). The average model distance between the two for all $18 \mathrm{~km}$ was $3.82 \mathrm{~cm}$ RMSE. 
Table 1. Performance metrics for OLE elements point cluster extraction

\begin{tabular}{ccccccccccccc}
\hline \multicolumn{4}{c}{ Performance metrics for C section extraction } & \multicolumn{5}{c}{ Performance metrics for cables extraction } \\
\hline Dataset & TP & FP & FN & Pr & R & F1 & TP & FP & FN & Pr & R & F1 \\
\hline D1 & 104 & 0 & 2 & $100 \%$ & $98.1 \%$ & $99.0 \%$ & 101 & 1 & 4 & $99.0 \%$ & $96.2 \%$ & $97.6 \%$ \\
D2 & 86 & 0 & 4 & $100 \%$ & $95.6 \%$ & $97.7 \%$ & 77 & 8 & 12 & $90.6 \%$ & $86.5 \%$ & $88.5 \%$ \\
D3 & 90 & 0 & 5 & $100 \%$ & $94.7 \%$ & $97.3 \%$ & 84 & 3 & 10 & $96.6 \%$ & $89.4 \%$ & $92.8 \%$ \\
\hline Average & & & $100 \%$ & $96.2 \%$ & $98.1 \%$ & & & & $95.6 \%$ & $91 \%$ & $93.2 \%$ \\
\hline
\end{tabular}
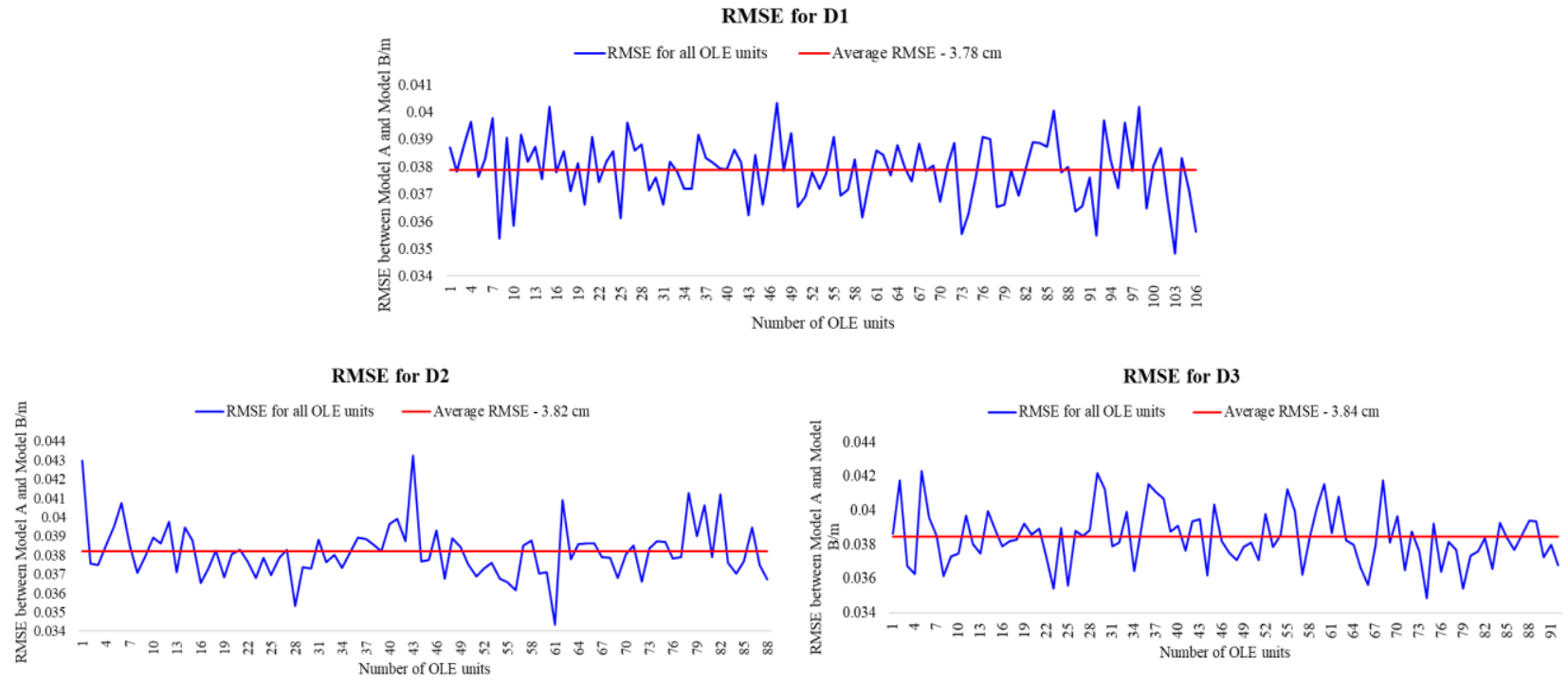

Figure 8. RMSE values for three datasets D1, D2 and D3

\section{Conclusions}

This paper presents a novel automated model-driven method that exploits the highly regulated and standardised nature of railways to generate gDTs of OLE elements for existing railways from PCD. Compared to existing methods, the proposed method is more consistent, less liable to human errors. The method was tested on an $18 \mathrm{~km}$ railway point cloud and achieves overall detection rates of $98.1 \%$ (F1 score) and 93.2\% (F1 score) for point cluster detection of C sections and cables respectively. The accuracy of the automated gDTs of OLE elements was evaluated by minimising the mean Euclidean distance between the ground truth model and the automated one. The average RMSE of the model for all $18 \mathrm{~km}$ equals to $3.82 \mathrm{~cm}$.

\section{Acknowledgements}

We express our gratitude for Fugro NL Land B.V. who provided data for evaluation. The research leading to these results has received funding from the Cambridge Commonwealth, European \& International Trust and Bentley Systems UK Plc. We gratefully acknowledge the collaboration of all academic and industrial project partners. Any opinions, findings and conclusions or recommendations expressed in this material are those of the authors and do not necessarily reflect the views of the institutes mentioned above.

\section{References}

[1] K. B. Salling and S. Leleur, "Accounting for the inaccuracies in demand forecasts and construction cost estimations in transport project evaluation," Transp. Policy, vol. 38, pp. 8-18, 2015.

[2] S. Vick and I. Brilakis, "Road Design Layer Detection in Point Cloud Data for Construction Progress Monitoring," J. Comput. Civ. Eng., vol. 32, no. 5, 2018.

[3] P. E. D. Love, D. D. Ahiaga-Dagbui, and Z. Irani, "Cost overruns in transportation infrastructure projects: Sowing the seeds for a probabilistic theory of causation," Transp. Res. Part A Policy Pract., vol. 92, pp. 184-194, 2016.

[4] B. Fanning, C. M. Clevenger, M. E. Ozbek, and H. Mahmoud, "Implementing BIM on Infrastructure: Comparison of Two Bridge Construction Projects," Pract. Period. Struct. Des. Constr., vol. 20, no. 4, 2015. 
[5] C. Eastman, K. Liston, R. Sacks, and K. Liston, BIM Handbook. 2008.

[6] C. Furneaux and R. Kivvits., "BIM Implications for Government.," Constr. Innov., no. 5, pp. 1-43, 2008.

[7] M. R. M. F. Ariyachandra and I. Brilakis, "Understanding the challenge of digitally twinning the geometry of existing rail infrastructure," in 12th FARU International Research Conference (Faculty of Architecture Research Unit), 2019.

[8] M. R. M. F. Ariyachandra and I. Brilakis, "Detection of Railway Masts in Air-Borne LiDAR Data," J. Constr. Eng. Manag., 2020.

[9] Network Rail, "Catalogue of Network Rail Standards." 2018.

[10] L. Cheng, L. Tong, Y. Wang, and M. Li, "Extraction of Urban Power Lines from Vehicle-Borne LiDAR Data," Remote Sens., vol. 6, no. 4, pp. 3302-3320, 2014.

[11] L. Zhu and J. Hyyppa, "The use of airborne and mobile laser scanning for modeling railway environments in 3D," Remote Sens., vol. 6, no. 4, pp. 3075-3100, 2014.

[12] H. Guan, J. Li, Y. Zhou, Y. Yu, C. Wang, and C. Wen, "Automatic Extraction of Power Lines From Mobile Laser Scanning Data," pp. 918921, 2014.

[13] M. Cserép, P. Hudoba, and Z. Vincellér, "Robust Railroad Cable Detection in Rural Areas from MLS Point Clouds," Free Open Source Softw. Geospatial Conf. Proc., vol. 18, no. $1,2018$.

[14] H. Guan, Y. Yu, J. Li, Z. Ji, and Q. Zhang, "Extraction of power-transmission lines from vehicle-borne lidar data," Int. J. Remote Sens., vol. 37, no. 1, pp. 229-247, 2016.

[15] Y. Liu, Z. Li, R. Hayward, R. Walker, and H. Jin, "Classification of Airborne LIDAR Intensity Data Using Statistical Analysis and Hough Transform with Application to Power Line Corridors," 2009 Digit. Image Comput. Tech. Appl., no. May, pp. 462-467, 2009.

[16] G. Sohn, Y. Jwa, and H. B. Kim, "AUTOMATIC POWERLINE SCENE CLASSIFICATION and RECONSTRUCTION USING AIRBORNE LIDAR DATA," ISPRS Ann. Photogramm. Remote Sens. Spat. Inf. Sci., vol. 1, no. September, pp. 167-172, 2012.
[17] H. B. Kim and G. Sohn, "Point-based classification of power line corridor scene using random forests," Photogramm. Eng. Remote Sensing, vol. 79, no. 9, pp. 821-833, 2013.

[18] B. Guo, Q. Li, X. Huang, and C. Wang, "An improved method for power-line reconstruction from point cloud data," Remote Sens., vol. 8, no. 1, pp. 1-17, 2016.

[19] Y. Wang, Q. Chen, L. Liu, D. Zheng, C. Li, and K. Li, "Supervised Classification of Power Lines from Airborne LiDAR Data in Urban Areas," Remote Sens., vol. 9, no. 8, p. 771, 2017.

[20] M. Arastounia, "Automatic Classification of LiDAR Point Clouds in A Railway Environment," Univ. Twente, Netherlands, 2012.

[21] M. Neubert et al., "Extraction of railroad objects from very high resolution helicopterborne LIDAR and ortho-image data," 2008.

[22] S. Zhang, C. Wang, Z. Yang, Y. Chen, and J. Li, "Automatic Railway Power Line Extraction Using Mobile Laser Scanning Data," ISPRS Int. Arch. Photogramm. Remote Sens. Spat. Inf. Sci., vol. XLI-B5, no. July, pp. 615-619, 2016.

[23] Y. Jwa and G. Sohn, "A piecewise catenary curve model growing for 3D power line reconstruction," Photogramm. Eng. Remote Sensing, vol. 78, no. 12, pp. 1227-1240, 2012.

[24] L. A. F. Fernandes and M. M. Oliveira, "Corrigendum to 'Real-time line detection through an improved hough transform voting scheme' [Pattern Recognition 41 (1) 299-314] (DOI:10.1016/j.patcog.2007.04.003)," Pattern Recognit., vol. 41, no. 9, p. 2964, 2008.

[25] E. Pastucha, "Catenary system detection, localization and classification using mobile scanning data," Remote Sens., vol. 8, no. 10, 2016.

[26] A. Sánchez-Rodríguez, M. Soilán, M. Cabaleiro, and P. Arias, "Automated inspection of Railway Tunnels' power line using LiDAR point clouds," Remote Sens., vol. 11, no. 21, 2019.

[27] Eurostat, "Railway transport - length of lines, by number of tracks," 2019.

[28] F. Tarsha-Kurdi, T. Landes, P. Grussenmeyer, and M. Koehl, "Model-driven and data-driven approaches using LIDAR data: Analysis and comparison," PIA 2007 - Photogramm. Image Anal. Proc., pp. 87-92, 2007. 